\title{
Complete thoracoscopic ablation of the left atrium via the left chest for treatment of lone atrial fibrillation
}

\author{
Ju Mei, MD, PhD, Nan Ma, MD, PhD, Fangbao Ding, MD, PhD, Yin Chen, MD, Zhaolei Jiang, MD, \\ Fengqing $\mathrm{Hu}, \mathrm{MD}$, and Haibo Xiao, MD, PhD
}

\begin{abstract}
Objective: We developed a new thoracoscopic ablation procedure for lone atrial fibrillation (AF) based on new endoscopic technology and the adoption of new types of energy.

Methods: Fifty-five patients with lone atrial fibrillation underwent this therapy. Patient age ranged from 30 to 81 years and there were 39 men and 16 women. Of these patients, 38 had paroxysmal atrial fibrillation, 14 had persistent atrial fibrillation, and 3 had longstanding atrial fibrillation. The procedure was performed on the beating heart through 3 ports in the left chest wall. Pulmonary vein isolation and ablation of the left atrium were achieved by bipolar radiofrequency ablation. Ganglionic plexus ablation was completed using the ablation pen. The left atrial appendage was excluded.
\end{abstract}

Results: Mean procedure duration was $106.6 \pm 42.8$ minutes. No conversion to sternotomy or pacemaker implantation occurred and no patients died. Their hospital stay was $5.3 \pm 2.0$ days with a mean follow-up of $12.6 \pm 2.2$ months. Forty-nine of 55 patients $(89.1 \%)$ patients were in sinus rhythm. Six patients could not maintain sinus rhythm. Thrombus in the left atrium and stenosis of the pulmonary vein were not found postoperatively.

Conclusions: This less invasive procedure proved to be safe and presented optimistic outcomes, so it deserves to be promoted as a treatment for lone atrial fibrillation. (J Thorac Cardiovasc Surg 2014;147:242-6)

Atrial fibrillation (AF) affects more and more patients in modern society, ${ }^{1}$ and leads to increased incidence of stroke, heart failure, and mortality. ${ }^{2,3}$ Improving recovery from AF is a hot topic. Maintaining sinus rhythm by antiarrhythmic drugs is difficult. ${ }^{4}$ The efficacy of catheter ablation is also unsatisfactory and associated with serious complications. ${ }^{5}$ The Cox Maze III procedure has the top success rate of $95 \%$ but it has not been widely adopted for the complexity of incisions and suturing. ${ }^{6}$ The problem is solved in part by the emergence of new types of energy, such as radiofrequencies, microwaves, freezing, and so forth. ${ }^{7}$ The Maze III procedure modified by the replacement of energy is adopted for its satisfactory recovery rate, but considerable concomitant trauma needs to be lessened, especially for patients with lone AF.

Wolf and colleagues ${ }^{8}$ introduced a surgical treatment for lone AF through an epicardial approach on the beating heart by thoracoscopic technique. The main components of this procedure are bilateral pulmonary vein isolation (PVI) using a bipolar radiofrequency ablation device, resection of

From the Department of Cardiothoracic Surgery, Xinhua Hospital, School of Medicine, Shanghai Jiaotong University, Shanghai, P. R. China.

Disclosures: Authors have nothing to disclose with regard to commercial support.

Received for publication July 31, 2012; revisions received Sept 24, 2012; accepted for publication Oct 2, 2012; available ahead of print Nov 5, 2012

Address for reprints: Ju Mei, MD, PhD, Department of Cardiothoracic Surgery, Xinhua Hospital, School of Medicine, Shanghai Jiao Tong University, 1665 Kong Jiang Road, Shanghai 200092, P. R. China (E-mail: ju_mei@yahoo.cn). $0022-5223 / \$ 36.00$

Copyright $₫ 2014$ Published by Elsevier Inc. on behalf of The American Association for Thoracic Surgery

http://dx.doi.org/10.1016/j.jtcvs.2012.10.005 the left atrial appendage, ablation of the ganglionic plexus (GP), and division of the ligament of Marshall. After treatment, about $90 \%$ patients are free of $\mathrm{AF}$ and accompanying trauma is reduced compared with previous procedures. ${ }^{8}$ The procedure of Wolf and colleagues ${ }^{8}$ requires $21-\mathrm{cm}$ ports and $15-\mathrm{cm}$ incision in both sides of the chest wall for the camera and ablation device. Yilmaz and associates ${ }^{9}$ developed a procedure for thoracoscopic ablation in 2007. The $5-\mathrm{cm}$ incision is replaced by 2 ports. The procedure becomes completely thoracoscopic; however, it still requires the ports on both sides of the chest wall, and ablation of the left atrium cannot be achieved. We introduce a less invasive procedure that allows for ablation of bilateral pulmonary veins, resection of the left atrial appendage, GP ablation, and ablation of the left atrium using a bipolar radiofrequency clamp that requires only 3 ports in the left chest wall.

\section{METHODS}

\section{Patient Selection}

Between October 2010 and June 2012, 55 patients with lone AF underwent the procedure. The study protocol was approved by the institutional review board, and informed consent was received from all patients. Patients with symptomatic AF between 30 years and 81 years of age were selected on the basis of the Heart Rhythm Society/European Heart Rhythm Association/European Cardiac Arrhythmia Society Expert Consensus Statement and the following inclusion criteria ${ }^{10}$ : drug-refractory AF, failed catheter ablation, history of stroke or embolism, or left ventricular ejection fraction of $30 \%$ or more. Previous cardiac or lung surgery, and a left atrium $>70$ $\mathrm{mm}$ were relative contraindications. Patient characteristics are detailed in Table 1. Preoperative AF-related histories are listed in Table 2. 

Abbreviations and Acronyms
$\mathrm{AF}=$ atrial fibrillation
$\mathrm{GP}=$ ganglionic plexus
$\mathrm{PVI}=$ pulmonary vein isolation

\section{Surgical Technique}

All 55 procedures were performed by Dr Mei after general anesthesia was administered to the patient with a double-lumen endotracheal tube. Transesophageal echocardiogram was performed in the operating room to verify the absence of left atrial thrombus. During the procedure, the patient was positioned in the right lateral decubitus position. The procedure was performed through 3 ports around the subscapular angle line of the left chest wall. The camera port $(10 \mathrm{~mm})$ was introduced in the eighth intercostal space at the subscapular angle line. The first working port (25 $\mathrm{mm}$ ) was introduced about $30 \mathrm{~mm}$ anterior to the subscapular angle line in the sixth intercostal space. The second working port $(10 \mathrm{~mm})$ was introduced $30 \mathrm{~mm}$ posterior to the subscapular angle line in the seventh intercostal space (Figure 1, A). The pericardium was opened a few centimeters anterior to the descending aorta after the dissection of ligament of inferior lung. The bilateral pulmonary veins and the posterior left atrium were exposed after the suspension of the pericardium. Blunt dissection around the pulmonary veins was performed using the AtriCure Lumitip Dissector (AtriCure, Inc, West Chester, Ohio) and PVI was achieved by bipolar radiofrequency ablation with the AtriCure Isolator Synergy ablation clamp (AtriCure, Inc). At least 10 times overlapping ablation lesions were performed at the antrum of the right and left pulmonary vein (Figure 1, $B$ and $C$ ). Electrical isolation of all pulmonary veins was tested using the AtriCure Synergy ablation pen (AtriCure, Inc). The linear lesion from the left inferior pulmonary vein to the right superior pulmonary vein could also be made by clamp after isolating the top of the left atrium (Figure 1,D). Other linear lesions on the left atrium were made using the AtriCure Synergy ablation pen. Linear lesions of the left atrium included left pulmonary vein to incision of left atrial appendage, left inferior pulmonary vein to the mitral valve annulus. The ablation of 3 circles and 2 lines on the left atrium were undertaken easily by direct vision (Figure 1,E). The ablation of the GP on the epicardium was also made using the AtriCure Isolator Synergy ablation pen. The left atrial appendage was excluded using an EZ 45 stapler (Ethicon EndoSurgery, Inc, Cincinnati, Ohio) (Figure 1, F). After these steps, cardioversion was performed immediately if AF did not stop.

\section{Postoperative Medical Management}

Amiodarone was administered to patients at $200 \mathrm{mg} /$ day for 3 months after the procedures and was then tapered in the presence of a stable sinus rhythm. Postoperative anticoagulation by warfarin or aspirin followed the score of $\mathrm{CHA}_{2} \mathrm{DS}_{2}-\mathrm{VAS}_{2}$ (Congestive Heart Failure, Hypertension, Age $\geq 75$ years [double], Diabetes mellitus, Stroke [double], Vascular disease, age 65-74, sex category [female]) and HAS-BLED (Hypertension, Abnormal renal and liver Function, Stroke, Bleeding, Labile INRs, Elderly, Drugs or alcohol). Anticoagulant drugs were discontinued if sinus rhythm was present 3 months after the procedure. Electrical cardioversion was performed if a patient had symptomatic $\mathrm{AF}<48$ hours or was anticoagulated adequately.

\section{Follow-up}

Baseline characteristics, and in-hospital and follow-up data were collected from clinical and outpatient clinic files. Questionnaires or telephone calls were used for follow-up. Heart rhythm documentation was based on electrocardiograms and 24-hour Holter (Royal Dutch Philips Electronics Ltd, Amsterdam, The Netherlands) monitoring analysis. Transthoracic echo and 24-hour Holter monitoring were evaluated at discharge and 1 , 3,6 , and 12 months postoperatively. The procedure was considered to be unsuccessful if an episode of $\mathrm{AF}$ (of a duration $>30$ seconds independent of amount of episodes or symptoms) occurred after a blanking period of 3 months.

\section{Statistical Analysis}

Data are presented as mean \pm standard deviation.

\section{RESULTS}

The procedure was completed successfully for all patients. The acute procedural success rate was $100 \%$. There was no conversion to sternotomy or thoracotomy. The average procedure time was $106.6 \pm 42.8$ minutes (range, 81-239 minutes). Eleven patients (20.0\%) received cardioversion after ablation in the operation room. One patient had failed electrical cardioversion. Mean bleeding was about $50 \mathrm{~mL}$ and no patient received blood products intraoperatively or during hospitalization. There were no mortalities in this population. No patient required pacemaker insertion. Two patients had onset of sinus tachycardia after the procedure. The sinus tachycardia stopped on its own and sinus rhythm was maintained until the data were collected. No obvious complications occurred after the procedures. Two patients (4\%) had early AF recurrence after cardioversion. Cardioversion was performed on them before discharge. One of them had failed electrical cardioversion; the other was successful. There was another patient who still had paroxysmal AF. Patients were discharged after a mean hospitalization of $5.3 \pm 2.0$ days (range, 4-14 days). Fifty-two patients $(94.5 \%)$ maintained a sinus rhythm when they were discharged. In-hospital results are summarized in Table 3. One patient was readmitted 4 weeks after discharge for pleural effusion and was treated by thoracostomy drain and oral steroid administration.

The mean follow-up duration was $12.6 \pm 2.2$ months. Forty-nine of 55 patients were free of AF, as confirmed by electrocardiograms or 24-hour Holter monitoring. All were asymptomatic. The 1-year success rate was $89.1 \%$. One patient, discharged with AF, who could not maintain a sinus rhythm before the procedure by oral amiodarone administration, recovered a sinus rhythm after the use of amiodarone 1 month later. Six patients ( 3 with paroxysmal AF and 3 with persistent/longstanding $\mathrm{AF}$ ) could not maintain a sinus rhythm after the procedure. Four of these 6 patients had early recurrence (1 patient with paroxysmal AF and 3 patients with persistent/longstanding $\mathrm{AF}$ ). Freedom from AF was $92.1 \%(35 / 38)$ and $82.4 \%(14 / 17)$ for paroxysmal $\mathrm{AF}$ and persistent/longstanding AF, respectively. Forty-nine patients evaluated beyond 3 months had been weaned successfully from antiarrhythmic drugs. Three months after the procedure, all computed tomographic and echocardiographic results were normal (no pulmonary vein stenosis or thrombus in the left atrium). 
TABLE 1. Patient characteristics

\begin{tabular}{lc}
\hline \multicolumn{1}{c}{ Characteristics } & Results \\
\hline Sex, male, \% (no. of males/no. of females) & $70.9(39 / 16)$ \\
Age, y; mean \pm SD & $59.2 \pm 15.4$ \\
Paroxysmal AF, n & 38 \\
Persistent AF, n & 14 \\
Longstanding persistent AF, n & 3 \\
NYHA class I, n & 37 \\
NYHA class II, n & 14 \\
NYHA class III, n & 4 \\
LA diameter, mm; mean \pm SD & $43.2 \pm 6.3$ \\
Duration of AF, y; mean \pm SD & $7.6 \pm 7.2$ \\
\hline$S D$, Standard deviation; $A F$, atrial fibrillation; $N Y H A$, New York Heart Association; \\
$L A$, left atrium.
\end{tabular}

\section{DISCUSSION}

Atrial fibrillation is the most common cardiac arrhythmia, but optimal therapy for AF has still not been discovered. The best aim of AF treatment is to achieve and maintain a sinus rhythm that results in fewer symptoms, decreased stroke risk and mortality, freedom from the antiarrhythmic drugs and anticoagulation, and an improved quality of life. ${ }^{11}$ The $95 \%$ sinus rhythm recovery rate reported by Cox is the top success rate, but few surgeons adopt the Cox Maze III procedure, which requires cardiopulmonary bypass and an arrested heart. ${ }^{12}$ The crossclamp time for a lone Cox Maze III procedure is about 60 to 120 minutes. ${ }^{12}$ Pacemakers are used in $15 \%$ of patients and the median length of stay is 9 to 12 days. Moreover, the procedure has significant morbidity. Catheter-based ablation is prevalent for its decreased pain and increased safety compared with the Maze III procedure. The deficiencies of this method include a limited success rate, a high incidence of recurrence, and associated serious complications such as stenosis of pulmonary veins, transesophageal fistulas, and so forth. ${ }^{13}$ It is inevitable that there are gaps created when performing a line of endocardial ablation dots, and the amount of energy applied during the procedure must be limited to avoid atrial perforation and transesophageal fistulas. The integrity and transmurality of

TABLE 2. Preoperative atrial fibrillation-related histories

\begin{tabular}{lc}
\hline \multicolumn{1}{c}{ Characteristics } & Results, $\mathbf{n}$ \\
\hline Hypertension & 38 \\
Diabetes mellitus & 14 \\
Stroke/arterial embolism & 5 \\
Alcoholic history & 7 \\
Smoking history & 23 \\
Catheter ablation & 7 \\
Permanent pacemaker implantation & 2 \\
Digoxin & 16 \\
Betaloc & 38 \\
Amiodarone & 40 \\
Aspirin & 42 \\
Warfarin & 30 \\
\hline
\end{tabular}

ablation cannot be guaranteed. ${ }^{14}$ Therefore, an ideal procedure should include a beating heart approach, intact transmural ablation, and minimal invasion.

The problems mentioned earlier were solved in part using the Wolf mini Maze procedure. Pulmonary vein isolation, GP ablation, and left atrial appendage amputation-the main components of the Wolf procedure-can also be done with our procedure. The intact, transmural isolation of pulmonary vein can be made by bipolar radiofrequency clamp on the beating heart. ${ }^{15}$ The excision of the left atrial appendage avoids production of thrombus to decrease effectively the incidence of stroke and embolism. ${ }^{16}$ The GP has an important influence on the generation and aggravation of AF. ${ }^{17}$ Scherlag and colleagues ${ }^{18}$ found that additional GP ablation had a better outcome in a prospective study that compared PVI with and without GP ablation. It was an encouraging result; however, the success rate should be further improved. About $90 \%$ of patients with paroxysmal AF are in normal sinus rhythm; patients with persistent and permanent $\mathrm{AF}$ are just $20 \%$ to $70 \%{ }^{14,19}$ Additional ablation should be undertaken for a greater recovery rate, especially for persistent and permanent AF.

Most spontaneous initiation of paroxysmal AF originates in the pulmonary veins. ${ }^{20}$ That is why the efficacy of PVI is higher in paroxysmal $\mathrm{AF}^{21}$ However, the posterior left atrium is also an important source that initiates and drives $\mathrm{AF}^{22}$ It should be more effective if we could remove the foci on the posterior left atrium with direct vision. In addition to those triggers stated earlier, change of substrate of atrial tissue is related strongly to persistent and permanent $\mathrm{AF}^{23}$ Therefore, only PVI, even with transmurality of ablation ensured and GPs ablation assisted, was not enough for persistent AF. It was inevitable that the success rate of treating persistent $\mathrm{AF}$ was poor. For these patients, we first need to make a more extensive linear lesion set, mimicking the Cox Maze III left atrial lesions, to prevent macro reentry. ${ }^{24,25}$ Second, we need to ensure the transmurality and continuity of the linear lesion. The linear lesion made using the bipolar radiofrequency clamp is currently the best way to ensure transmurality and continuity. ${ }^{15}$ Our approach allows accurate, transmural, and continuous linear lesion on the left atrium by using a bipolar radiofrequency clamp. It was the first time to expose the posterior left atrium from epicardium from left chest wall. The ablation of 3 circles and 2 lines on the left atrium were achieved easily with direct vision. Meanwhile, any foci on the posterior left atrium could also be ablated. Our data show a recovery rate this is no less than that of Wolf and colleagues. ${ }^{8}$ Whether a better recovery rate can be achieved must be confirmed by more cases and further follow-up.

A minimally invasive procedure is one direction for future surgery. Ablation from epicardium enables this procedure to be performed on a beating heart without cardiopulmonary bypass. The sternotomy or rib-spreading 

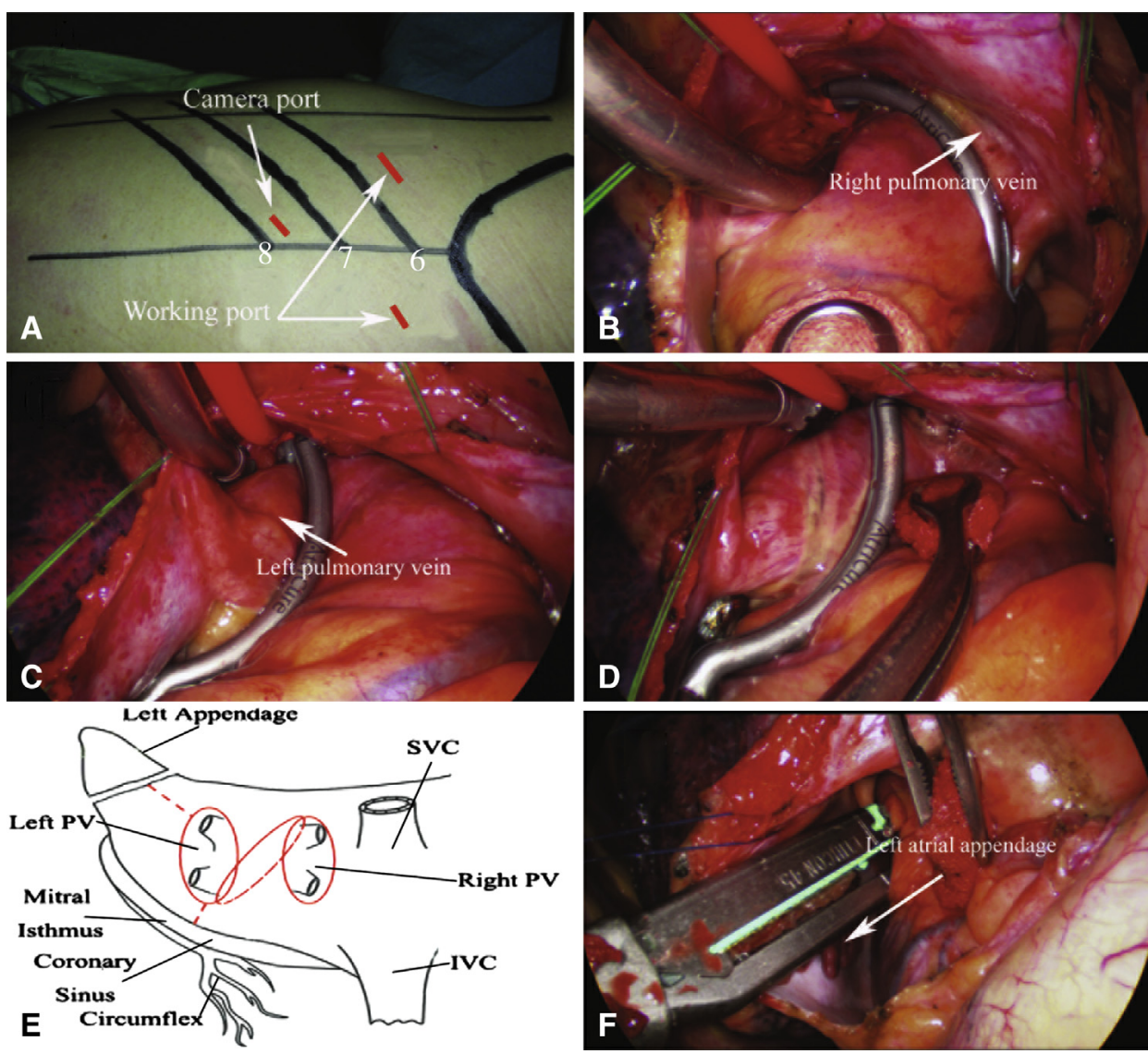

FIGURE 1. Complete thoracoscopic ablation of the left atrium. (A) The entire procedure is performed thoracoscopically through 3 ports on the left chest wall. The black lines with numbers indicate the intercostal spaces. The red lines show the position of the ports. (B) The bipolar radiofrequency ablation clamp is placed around the left pulmonary vein. (C) The bipolar radiofrequency ablation clamp is placed around the right pulmonary vein. (D) The linear lesion from the left inferior pulmonary vein to the right superior pulmonary vein by the bipolar radiofrequency ablation clamp. (E) The ablation of 3 circles and 2 lines on the left atrium. (F) Amputation of the left atrial appendage with a stapler. $P V$, Pulmonary vein; $S V C$, superior vena cava; $I V C$, inferior vena cava.

thoracotomy is obviated by our approach. Our approach is the first to ablate the bilateral pulmonary veins via the left chest wall. In addition, it is easier to perform than previous surgical procedures. Therefore, we have reason to believe our procedure will be widely adopted for its efficacy, safety, convenience, and minimal invasion.

TABLE 3. Patient outcomes

\begin{tabular}{lc}
\hline \multicolumn{1}{c}{ Outcome } & Results \\
\hline Mortality, $\mathrm{n}$ & 0 \\
Procedure time, min & $106.6 \pm 42.8$ \\
Conversion to sternotomy, $\mathrm{n}$ & 0 \\
Cardioversion in OR, $\mathrm{n}$ & 11 \\
Cardioversion before discharge, $\mathrm{n}$ & 2 \\
Discharged with AF, $\mathrm{n}$ & 3 \\
Hospital stay, d & $5.3 \pm 2.0$ \\
Recurrence, $\mathrm{n}$ & 6 \\
Sinus rhythm without AADs, $\mathrm{n}$ & 49 \\
\hline
\end{tabular}

$O R$, Operating room; $A F$, atrial fibrillation; $A A D s$, antiarrhythmic drugs.
We thank Dr Saie Shen, Dr Yuan Sun, Dr Yin Cai, Dr Mingsong Wang, and Huihua Chen for their excellent experimental and clinical support.

\section{References}

1. Zhou Z, Hu D. An epidemiological study on the prevalence of atrial fibrillation in the Chinese population of mainland China. J Epidemiol. 2008;18:209-16.

2. Kimura K, Minematsu K, Yamaguchi T. Atrial fibrillation as a predictive factor for severe stroke and early death in 15,831 patients with acute ischaemic stroke. J Neurol Neurosurg Psychiatry. 2005;76:679-83.

3. Savelieva I, Camm AJ. Atrial fibrillation and heart failure: natural history and pharmacological treatment. Europace. 2004;5:5-19.

4. Kalus JS. Pharmacologic management of atrial fibrillation: established and emerging options. J Manage Care Pharm. 2009;15:S10-8.

5. Angkeow P, Calkins HG. Complications associated with radiofrequency catheter ablation of cardiac arrhythmias. Cardiol Rev. 2001;9:121-30.

6. Cox JL, Ad N, Palazzo T, Fitzpatrick S, Suyderhoud JP, DeGroot KW, et al. Current status of the Maze procedure for the treatment of atrial fibrillation. Semin Thorac Cardiovasc Surg. 2000;12:15-9.

7. Aktas MK, Daubert JP, Hall B. Surgical atrial fibrillation ablation: a review of contemporary techniques and energy sources. Cardiol J. 2008;15:87-94.

8. Wolf RK, Schneeberger EW, Osterday R, Miller D, Merrill W, Flege JB Jr, et al. Video-assisted bilateral pulmonary vein isolation and left atrial appendage exclusion for atrial fibrillation. J Thorac Cardiovasc Surg. 2005;130:797-802. 
9. Yilmaz A, Geuzebroek GS, Van Putte BP, Boersma LV, Sonker U, De Bakker JM, et al. Completely thoracoscopic pulmonary vein isolation with ganglionic plexus ablation and left atrial appendage amputation for treatment of atrial fibrillation. Eur J Cardiothorac Surg. 2010;38:356-60.

10. Calkins H, Kuck KH, Cappato R, Brugada J, Camm AJ, Chen SA, et al. 2012 HRS/EHRA/ECAS expert consensus statement on catheter and surgical ablation of atrial fibrillation: recommendations for patient selection, procedural techniques, patient management and follow-up, definitions, endpoints, and research trial design. J Interv Card Electrophysiol. 2012;33:171-257.

11. Wolf PA, Mitchell JB, Baker CS, Kannel WB, D'Agostino RB. Impact of atrial fibrillation on mortality, stroke, and medical costs. Arch Intern Med. 1998;158: 229-34.

12. Prasad SM, Maniar HS, Camillo CJ, Schuessler RB, Boineau JP, Sundt TM III, et al. The Cox Maze III procedure for atrial fibrillation: long-term efficacy in patients undergoing lone versus concomitant procedures. J Thorac Cardiovasc Surg. 2003;126:1822-8.

13. Cappato R, Calkins H, Chen SA, Davies W, Iesaka Y, Kalman J, et al. Worldwide survey on the methods, efficacy, and safety of catheter ablation for human atrial fibrillation. Circulation. 2005;111:1100-5.

14. Castellá M, Pereda D, Mestres CA, Gómez F, Quintana E, Mulet J. Thoracoscopic pulmonary vein isolation in patients with atrial fibrillation and failed percutaneous ablation. J Thorac Cardiovasc Surg. 2010;140:633-8.

15. Gillinov AM, McCarthy PM. Atricure bipolar radiofrequency clamp for intraoperative ablation of atrial fibrillation. Ann Thorac Surg. 2002;74:2165-8.

16. Gillinov M. Advances in surgical treatment of atrial fibrillation. Stroke. 2007;38: 618-23.
17. Patterson E, Po SS, Scherlag BJ, Lazzara R. Triggered firing in pulmonary veins initiated by in vitro autonomic nerve stimulation. Heart Rhythm. 2005; 2:624-31.

18. Scherlag BJ, Nakagawa H, Jackman WM, Yamanashi WS, Patterson E, Po S, et al. Electrical stimulation to identify neural elements on the heart: their role in atrial fibrillation. J Interv Card Electrophysiol. 2005;13:37-42.

19. Beyer E, Lee R, Lam BK. Point: minimally invasive bipolar radiofrequency ablation of lone atrial fibrillation: early multicenter results. J Thorac Cardiovasc Surg. 2009;137:521-6.

20. Haïssaguerre M, Jaï P, Shah DC, Takahashi A, Hocini M, Quiniou G, et al. Spontaneous initiation of atrial fibrillation by ectopic beats originating in the pulmonary veins. $N$ Engl J Med. 1998;339:659-66.

21. Cui YQ, Li Y, Gao F, Xu CL, Han J, Meng X, et al. Video-assisted minimally invasive surgery for lone atrial fibrillation: a clinical report of 81 cases. $J$ Thorac Cardiovasc Surg. 2010;139:326-32.

22. Voeller RK, Bailey MS, Zierer A, Lall SC, Sakamoto S, Aubuchon K, et al. Isolating the entire posterior left atrium improves surgical outcomes after the Cox Maze procedure. J Thorac Cardiovasc Surg. 2008;135:870-7.

23. Burstein B, Nattel S. Atrial fibrosis: mechanisms and clinical relevance in atrial fibrillation. J Am Coll Cardiol. 2008;51:802-9.

24. Geuzebroek GS, Ballaux PK, van Hemel NM, Kelder JC, Defauw JJ. Mediumterm outcome of different surgical methods to cure atrial fibrillation: is less worse? Interact Cardiovasc Thorac Surg. 2008;7:201-6.

25. Wisser W, Seebacher G, Fleck T, Aigner C, Khazen C, Stix G, et al. Permanent chronic atrial fibrillation: is pulmonary vein isolation alone enough? Ann Thorac Surg. 2007;84:1151-7. 